\title{
The Silicon Upstream Tracker for the LHCb Upgrade
}

\author{
Nicola Neri* \\ INFN, Sezione di Milano, Milano, Italy \\ E-mail: nicola.neri@mi.infn.it
}

The detector for the $\mathrm{LHCb}$ upgrade is designed for $40 \mathrm{MHz}$ readout (the maximum beam-crossing rate), allowing the experiment to collect $5 \mathrm{fb}^{-1}$ per year. The upgrade of the Upstream Tracker, upstream of the magnet, is crucial for charged track reconstruction and fast trigger decisions based on a tracking algorithm involving also vertex detector information. The detector consists of 4 planes made of single sided silicon strip sensors: the two external planes are sensitive to the horizontal coordinate while the others are tilted by $\pm 5^{\circ}$. The radiation dose integrated in 10 years is estimated to be $35 \mathrm{MRad}$ for the inner region; the sensor operating temperature will be $-{ }^{\circ} 5$ to prevent sensor thermal runaway. The material budget has to be minimized, imposing severe challenges to the design of the support structure, cooling system and electronics in the active area. We will present here the conceptual design of the detector and the status of the R\&D for the project.

22nd International Workshop on Vertex Detectors

September 15th-20th, 2013

Lake Starnberg, Germany

\footnotetext{
*Speaker.

on behalf of the UT collaboration for the LHCb upgrade
} 


\section{Introduction}

Heavy flavor physics has a great discovery potential that in principle can extend the search for new particles beyond the energy scale of the LHC. This is possible through indirect searches for new physics, as opposite to direct searches, where new particles can be discovered by reconstructing their invariant masses. In indirect searches, the sensitivity to new physics arises from loop diagrams where new particles contribute with sizable effects to physics observables. Experimental results have to be compared with theoretically "clean" predictions, and high precision measurements are crucial for the success of this scientific program. In this respect, there are many "theory clean" measurements currently limited by the statistical uncertainty that will benefit of a much larger data sample [1].

The LHCb detector [2] is a single-arm forward spectrometer covering the pseudorapidity range $2<\eta<5$, designed for the study of particles containing $b$ or $c$ quarks. The results from LHCb, based on data collected during the LHC Run 1, have definitively proved that precision measurements in the heavy-flavour sector can be made in the extreme environment of high-energy $p p$ collisions. The readout and trigger scheme of the current $\mathrm{LHCb}$ detector, however, limit the amount of luminosity that can be recorded, and therefore the precision that can be achieved. In order to overcome these limitations, it is necessary to upgrade the current detector to $40 \mathrm{MHz}$ readout with a highly flexible trigger. The trigger scheme for the upgrade consists of a Low-Level Trigger (LLT), based on the information of the electromagnetic calorimeter and of the muon detector, and a HighLevel Trigger (HLT), a software-based trigger running on a CPU farm that profits from the fast tracking capability of the upgraded LHCb detector. The LLT will serve as a throttle, providing the trigger bandwidth $(1-40 \mathrm{MHz})$ to be adapted to the available CPU power. The output data rate of the HLT for the offline event reconstruction will be $20 \mathrm{kHz}$. The LHCb experiment, after its upgrade foreseen in 2020, will be able to integrate $50 \mathrm{fb}^{-1}$ in 10 years of data taking with instantaneous luminosity of $2 \times 10^{33} \mathrm{~cm}^{-2} \mathrm{~s}^{-1}$ at regime. The data rate will be increased substantially, as well as the trigger efficiency, leading to improvements in annual signal yields, compared to those obtained in 2011, by a factor of around ten for muonic $B$ decays and twenty or more for heavy-flavour decays to hadronic final states.

\section{Overview of UT detector}

The LHCb tracking system consists of the Vertex Locator (VELO), the Tracker Turicensis (TT), located in front of a $4 \mathrm{Tm}$ dipole magnet, and and a combination of silicon strip detectors (Inner Tracker, IT) and straw drift chambers (Outer Tracker, OT) placed behind. The VELO is a silicon strip device positioned close to the interaction region that measures the locations of the $p p$ interaction primary vertices and the locations of the decay vertices of long-lived particles. The TT, the OT and IT are responsible for the tracking of charged particles, and for the measurement of their momenta. For the LHCb upgrade, all the tracking detectors will be replaced: the VELO will be based on pixel sensor technology, the Upstream Tracker (UT) will replace the TT and the Scintillating Fiber Tracker (SciFi), based on scintillating plastic fibers, will replace the OT and IT detectors. The tracking system for the LHCb upgrade is sketched in Fig. 1. The UT detector upgrade main goals are: (i) enable $40 \mathrm{MHz}$ readout, (ii) enable full coverage of tracks within the geometric ac- 


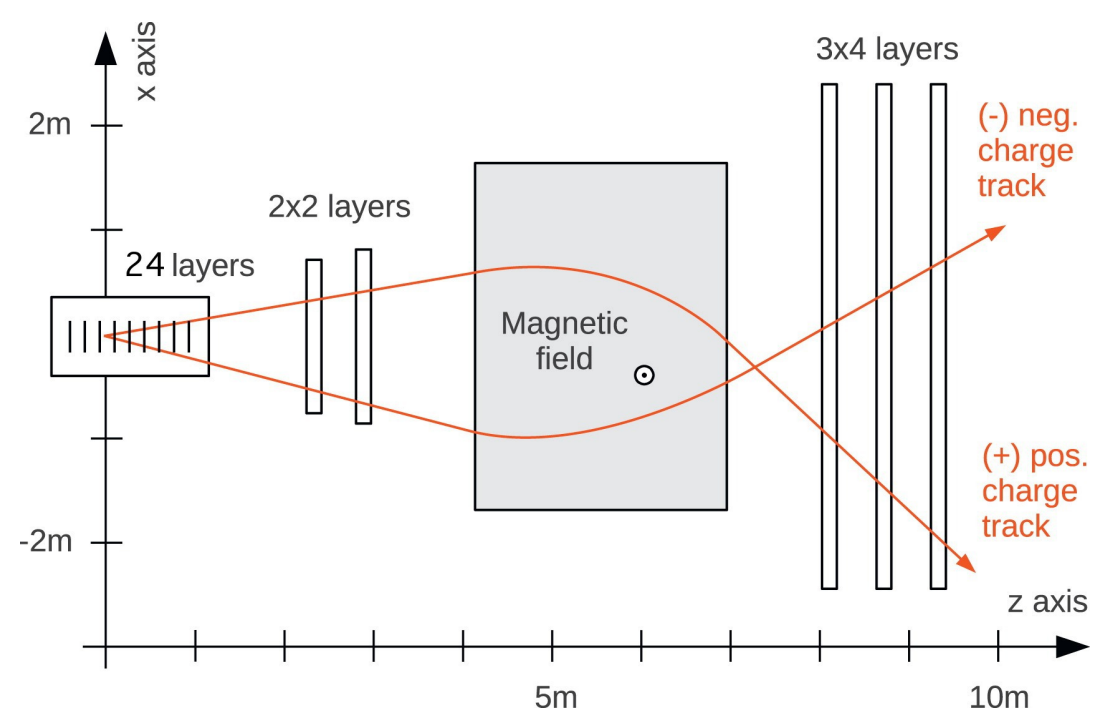

Figure 1: Schematic representation of the tracking system for the LHCb upgrade. The interaction region is inside the VELO at $z \simeq 0$, the UT is positioned before the magnet, and the SciFi is behind it. The main component of the field of the dipole magnet is parallel to the $y$-axis. The curved lines represent trajectories of a positively and negatively charged particle.

ceptance, (iii) increase the small angle acceptance, (iv) improve the sensor segmentation, and (v) minimize the detector material in terms of radiation length. The UT detector consists of 4 planes of single sided silicon strip sensors, positioned upstream of the magnet at about $2.27-2.70 \mathrm{~m}$ from the interaction region. The silicon layers are arranged in a $(x-u-v-x)$ configuration, where the $x$ planes measure the $x$ coordinate and the silicon strips runs along the $y$ direction, while the $u$ and the $v$ planes are tilted of $+5^{\circ}$ and $-5^{\circ}$ with respect to the $y$ direction. The spectrometer acceptance outer limit is $\pm 300 \mathrm{mrad}$ in the bending plane $(x z)$ and $\pm 250 \mathrm{mrad}$ in the plane perpendicular to that $(y z)$, while the inner limit is about $10 \mathrm{mrad}$ determined by the beam pipe radius. The detector typical acceptance corresponds to a pseudorapidity range of $2<\eta<5$, which is covered with a nominal fiducial area of $1,538 \times 1,346 \mathrm{~mm}^{2}\left(1,731 \times 1,346 \mathrm{~mm}^{2}\right)$ for the most upstream (downstream) planes.

The silicon strip sensors are organized in a vertical column structure, the "stave", which provides the mechanical support and the cooling for the front-end electronics and the sensors, which will be operated at a temperature of $-5^{\circ}$. The two planes closest to the interaction region consist of 16 staves and the remaining two planes are made of 18 staves. These are mounted adjacent to one another, and overlaps between the sensors ensure full coverage. The layers are mounted on a C-shaped aluminium frame outside of the spectrometer acceptance and inside a detector box, with thin windows at the front and back faces, to ensure thermal and electrical insulation. The SALT chips are bonded directly to the sensors and are positioned in the active tracking volume. $\mathrm{A} \mathrm{CO}_{2}$ bi-phase cooling system will provide efficient cooling with reduced impact on the material budget. Data are transferred to the periphery via thin flex cables, and separate flex cables bring the power to the sensors and the electronics. 


\section{UT objectives and expected performance}

The $\mathrm{LHCb}$ detector proved to be able to cope with a larger number of visible interactions per bunch-crossing, $\mu$, than originally planned $(\mu=0.4$ ). In early 2010 , due to a low number of bunches in the LHC, it was reached $\mu=2.5$, similar to the value expected for the LHCb upgrade. However, an upgraded LHCb detector will experience larger bunch-to-bunch spillover, due to the $25 \mathrm{~ns}$ bunch spacing, and higher track multiplicities, given the expected increase of the LHC beam energies. Extensive studies based on Monte Carlo simulations were performed trying to reproduce the upgrade conditions, e.g. upgraded detector, number of primary vertices, and track multiplicity. Maximal tracking efficiency with minimal rate of fake tracks represents the challenge of the UT detector design at high luminosity and which determined its specifications.

The UT is a crucial element in track reconstruction whose main objectives are: (i) reduce ghost tracks ${ }^{1}$, (ii) reconstruction of long-live particles decaying after the VELO, e.g. $K_{\mathrm{S}}^{0} \rightarrow \pi^{+} \pi^{-}$and $\Lambda \rightarrow p \pi^{-}$, (iii) provide $p_{\mathrm{T}}$ estimate for charged tracks and reject low momentum tracks for faster track reconstruction for trigger decisions (HLT). In Fig. 2, the ghost track probability is shown as a function of the transverse momentum, $p_{\mathrm{T}}$, for charged tracks transversing all the tracking system ("long" tracks) originated from inclusive $b$ events. The ghost track probability is significantly reduced once UT hits are required for the track reconstruction. Ghost track reduction is crucial for speeding up trigger timings and for background suppression in physics analyses. At LHCb, most of the $K_{\mathrm{S}}^{0}$ decay after VELO, and the UT information is especially important for their reconstruction. According to Monte Carlo simulation studies, $73 \%$ of $\bar{B}^{0} \rightarrow J / \psi K_{\mathrm{S}}^{0}$ events are reconstructed from $K_{\mathrm{S}}^{0}$ decays downstream of the VELO, using UT and SciFi hits only. In Table 3, the HLT timing results are shown using an emulation of the trigger system for the LHCb upgrade. The results have to be compared with the allowed timing budget for the upgraded trigger farm of about $16 \mathrm{~ms}$ per event, on average ${ }^{2}$. Using VELO and UT information only, it is possible to reconstruct track segments (VELO-UT tracks) and estimate their momenta. The average momentum resolution for VELO-UT tracks is about $\sigma\left(p_{\mathrm{T}}\right) / p_{\mathrm{T}} \sim 15 \%$, in the $p_{\mathrm{T}}$ range [0.1-10] $\mathrm{GeV} / c$. The bending power in the UT volume is limited to about $0.11 \mathrm{Tm}$. However, the momentum resolution is dominated by multiple scattering over almost the full range of particle momenta, hence the material budget has to be minimized. In order to reconstruct long tracks, a lower $p_{\mathrm{T}}$ cutoff of $400 \mathrm{MeV} / c$ is applied to VELO-UT tracks, before matching them with track segments reconstructed in the SciFi. The $p_{\mathrm{T}}$ cutoff and the use of the VELO-UT momentum estimate in the track matching algorithm, reduce significantly the combinatorics and the ghost track rate. The timing and the ghost track rate increase almost by a factor 3 when the $p_{\mathrm{T}}$ cutoff and the momentum estimate is not used in the forward tracking algorithm.

\section{Silicon sensors}

The sensor design has to satisfy the specifications imposed by the physics requirements and to

\footnotetext{
${ }^{1} \mathrm{~A}$ ghost track is a fake track. For example it can be formed by matching a real track segment in the VELO (VELO seed) with a real track segment in the downstream tracker (SciFi seed).

${ }^{2}$ It should be noted that the execution time is machine dependent. The timing results presented here are obtained with a machine that is about 3 times faster than a $2.8 \mathrm{GHz}$ Xeon.
} 


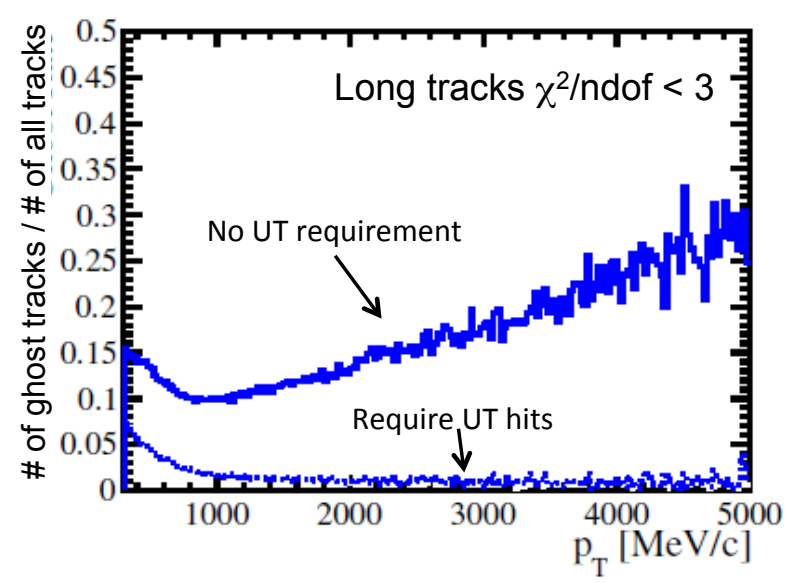

Figure 2: Probability for ghost tracks as a function of $p_{\mathrm{T}}$ for tracks inside the UT fiducial volume and with $\chi^{2} /$ ndof $>3$. For long tracks there is ghost contribution from matching with SciFi segments, which is significantly reduced by requiring UT hits.

\begin{tabular}{|l|c|}
\hline Tracking algorithm & Time $(\mathrm{ms})$ \\
\hline VELO tracking & 1.9 \\
VELO-UT tracking & 1.8 \\
Forward tracking & 4.4 \\
\hline Total HLT2 tracking & 8.1 \\
\hline
\end{tabular}

Table 1: Timing of the HLT2 tracking sequence with instantaneous luminosity of $2 \times 10^{33} \mathrm{~cm}^{-2} \mathrm{~s}^{-1}$. Tracks with $p_{\mathrm{T}}$ above $400 \mathrm{MeV} / c$ are searched for by the forward tracking algorithm.

cope with the radiation dose of about $35 \mathrm{MRad}$, expected in the innermost part of the detector with an integrated luminosity of $50 \mathrm{fb}^{-1}$. The hit density in the UT, $\Phi$, depends strongly on the radial distance $r$ from the interaction region, $\Phi(r) \sim 3.832 \cdot r^{-1.684}$ hits/event $\cdot \mathrm{cm}^{-2}$, and the radiation decreases by a factor of 10 at a radius greater than $15 \mathrm{~cm}$. Simulation studies have demonstrated that a single-hit resolution of about $50 \mu \mathrm{m}$ is adequate for UT. The momentum resolution of the spectrometer is then dominated by multiple scattering over almost the full range of particle momenta. Readout strip pitches of about $190 \mu \mathrm{m}$ would meet this requirement, however a pitch of about $95 \mu \mathrm{m}$ was chosen for the inner region to keep the maximum strip occupancy at the level of a few percent while minimizing the number of readout channels. For the same reason readout strip lengths of $97 \mathrm{~mm}$, in the outer region, and of $48 \mathrm{~mm}$, in the inner region, were chosen. In addition, Monte Carlo simulations have proved that having a poorer granularity in the central sensors would increase significantly the ghost rate of VELO-UT tracks. In the inner region, sensors with circular cut-out allow the beam pipe to pass through the UT while extending the detector acceptance at $\eta \sim 5$. A schematic of the sensor segmentation is shown in Fig. 3, where 4 types of sensor geometries are considered. The physics hole in the inner sensors has a radius of $33.4 \mathrm{~mm}$; it includes also the space for the thermal insulation $(5.0 \mathrm{~mm})$ and the clearance $(2.5 \mathrm{~mm})$ determined by the beam pipe specifications. The thermal insulation compresses to $3.5 \mathrm{~mm}$ when put in place and it is 
considered in the calculation. An additional radial space of $0.8 \mathrm{~mm}$ on the sensors is dedicated to the guard ring, hence the inner radius of the UT active area is at $34.2 \mathrm{~mm}$. The sensors are $250 \mu \mathrm{m}$ thick and are based on $n$-in- $p$ technology for the inner sensors with $95 \mu \mathrm{m}$ pitch; $p$-in- $n$ technology is currently the baseline solution for the outer sensors with $190 \mu \mathrm{m}$ pitch. The different technology choice for the outer sensors will be validated with radiation damage tests, system design and cost considerations. R\&D on technology choices and prototypes for the inner sensors have been initiated with 2 different vendors, i.e. Hamamatsu ${ }^{3}$ and Micron ${ }^{4}$.

The most probable charge released in the sensors will be about $20 \mathrm{ke}$ corresponding to a charge of $3.2 \mathrm{fC}$. A signal over noise ratio of about 20 is expected assuming an equivalent noise charge (ENC) of about $1000 e$ in the SALT chip and $10 \mathrm{pF}$ input capacitance to the charge sensitive preamplifier. The bulk generated current in radiation damaged sensors is the main source of the reverse bias current, $I_{R}$, which increases power consumption and introduces noise; it strongly dependent on temperature $T$,

$$
I_{R}(T) \propto T^{2} e^{-E / 2 k_{B} T}
$$

where $E \sim E_{g}=1.2 \mathrm{eV}$ and $k_{B}$ is the Boltzmann constant. In order to prevent thermal runaway of the silicon sensors in presence of radiation damage, they will be operated at a temperature of $-{ }^{\circ} 5$.

\section{Front-end electronics}

The SALT chip [3, 4] provides zero-suppressed digital signal with 6 bit ADC resolution and operates for both signal polarities. It is a 128 channel ASICS based on CMOS IBM $130 \mathrm{~nm}$ technology, which ensures good radiation hardness up to about $50 \mathrm{MRad}$. It features a charge-sensitive preamplifier, a shaper, a 6-bit ADC, a DSP block for zero suppression and data compression, and a data serializer. The chip is positioned in the tracking volume, close to the sensor and bonded directly to it, in order to minimize the input capacitance to the charge-sensitive preamplifier while improving the $\mathrm{S} / \mathrm{N}$. The shaper peaking time is about $25 \mathrm{~ns}$, with a fast baseline restore to limit the spillover signals, in the next beam-crossing 25 ns later, to a maximum of $5 \%$ of the signal. Particular attention in the design is devoted to the minimization of the power consumption, to be below $6 \mathrm{~mW} /$ channel, i.e. $0.77 \mathrm{~W} /$ chip. The first SALT chip prototype with 8 channels, featuring preamplifier and shaper and 2 channels of Single-to-Differential converter, has been designed and tested. Preliminary results are encouraging and within the specifications in terms of power consumption, fast baseline restore and noise. The prototype ASICS have been tested with negative and positive pulses and the gain of the preamplifier has been measured to be about $30 \mathrm{mV} / \mathrm{fC}$ in both cases using an input capacitance of $10 \mathrm{pF}$. When varying the input capacitance, the shaper peaking time changes from $23.5 \mathrm{~ns}$ at $0 \mathrm{pF}$ to $29 \mathrm{~ns}$ at $50 \mathrm{pF}$, while the preamplifier gain changes from $36 \mathrm{mV} / \mathrm{fC}$ at $0 \mathrm{pF}$ to $18 \mathrm{mV} / \mathrm{fC}$ at $50 \mathrm{pF}$. The measurement of the ENC is about $1000 e$ at $10 \mathrm{pF}$ and the ENC slope in the range $10-40 \mathrm{pF}$ is about $57 \mathrm{e} / \mathrm{pF}$. The next ASIC submission is scheduled in February 2014, where several issues coming from measurements of present prototypes will be addressed. A prototype ASICS with 8 channels of 6-bit successive approximation register (SAR) ADC, a multiplexing and serialization circuitry, a phase-locked loop (PLL) block [5] and a scalable

\footnotetext{
${ }^{3}$ Hamamatsu Photonics K.K., Japan.

${ }^{4}$ Micron Semiconductor Ltd, UK.
} 


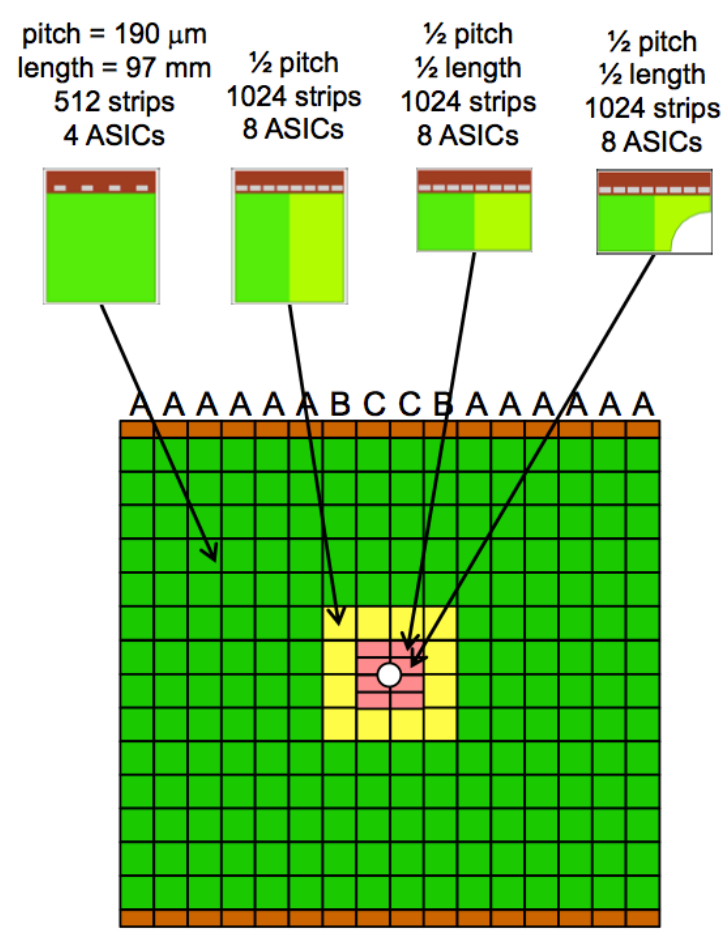

Figure 3: A schematic of the UT sensor segmentation. A strip pitch of about $190 \mu \mathrm{m}$ and strip length of about $97 \mathrm{~mm}$ are used in the outer region, a strip pitch of about $95 \mu \mathrm{m}$ and strip length of about $97 \mathrm{~mm}$ for the intermediate region, and sensors with a strip pitch of about $95 \mu \mathrm{m}$ and strip length of about $48 \mathrm{~mm}$ are used in the inner region.

low-voltage signalling (SLVS) input/output circuitry was fabricated and tested. First measurements are encouraging and showed that the ADC is working very well: good linearity at $50 \mathrm{MHz}$ sampling frequency (working fine also at $80 \mathrm{MHz}$ ), low power consumption, and effective number of bits between 5.7 and 5.9. The first results on the PLL and the SLVS blocks verified their functionality to be consistent with the specifications.

Hybrid flex circuits host the SALT chip and provide good thermal bridge for cooling and interconnections to low mass flex cables, based on kapton, with copper lines for data and power signals. Data lines will be connected to the GBT [6] boards providing transition from electrical to optical signals.

\section{Mechanics and cooling}

The mechanical requirements and design goals of UT [7] are summarized below. The UT conceptual design consists of 4 planes $(x-u-v-x)$ of single sided silicon strip detectors, tiled around the beam pipe and covering the acceptance, corresponding to the pseudorapidity range $2<\eta<5$ in the bending plane $(x z)$. The silicon sensors are arranged in vertical staves that are mounted adjacent to one another. Sensors overlap in $x$ is obtained by staggering the $z$ position of adjacent staves. 
The stave has a modular structure whose elementary unit is the "hybrid", represented in Fig. 4 (left plot). It consists of the sensor, the support structure, the ASICS, the hybrid flex circuits and the low mass flex cables for data and power signals. The sensors are tiled on both sides of the stave, as shown in Fig. 4 (right plot), allowing for the overlap in $y$. The design is inspired by the ATLAS IBL stave [8], with carbon fiber reinforced plastic (CFRP) facings, providing structural support, which are glued with epoxy to a carbon foam interior. The stave is a closed structure which
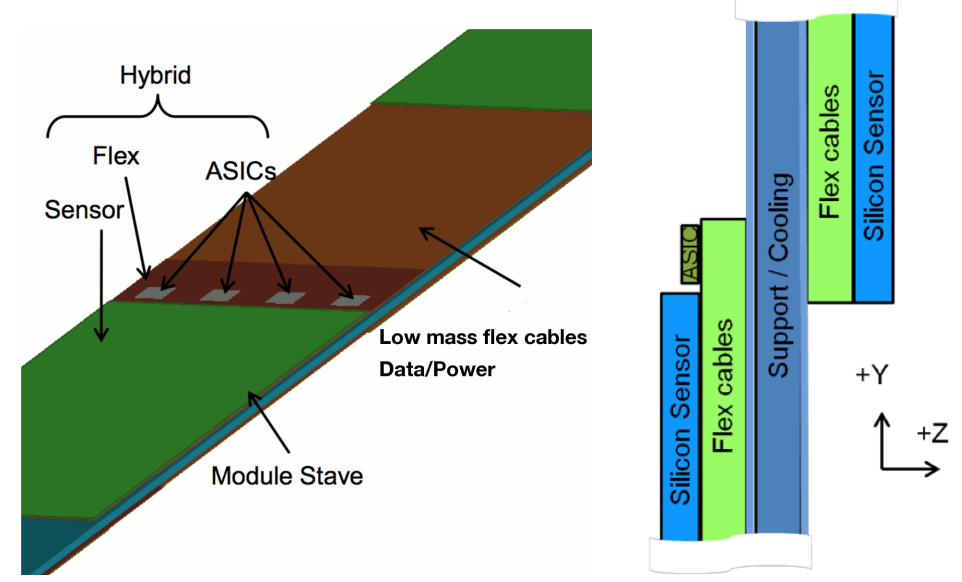

Figure 4: Left plot: sketch of the UT hybrid elementary module composed by the sensor, the support structure, the ASICS, the hybrid flex, and the low mass flex cables for data and power signals. Right plot: side view of the UT stave. The sensor and the front-end electronics alternates on both sides of the stave providing sensor overlap in the $y$ direction.

provides stiffness and the facings are separated using lightweight foam. The metal cooling tubes are embedded in the interior of the structure, as shown in Fig. 5, and the carbon foam provides good heat transfer. The tubes are thin, $2.2 \mathrm{~mm}$ outer diameter and $0.1 \mathrm{~mm}$ wall thickness, and are made of Titanium alloy. The cooling system has to maintain the temperature of the sensors at $-5^{\circ} \mathrm{C}$ by removing the heat generated in the ASICS, conservatively assumed to be $0.77 \mathrm{~W} / \mathrm{chip}$, and in the silicon sensors due to self-heating. The power consumption of UT has been estimated to be about $800 \mathrm{~W} /$ plane for a total of $3.2 \mathrm{~kW}$. A dedicated simulation study, based on finite element analysis, has proved that evaporative $\mathrm{CO}_{2}$ cooling is the optimal choice in terms of cooling efficiency and material budget. A "snake pipe" design with bent tubes passing underneath the ASICS, see Fig. 5, is currently considered as the baseline solution providing maximal heat transfer. However, several issues have still to be assessed, e.g. the tube bending radius, the thermal contractions and the mechanical deformations of the stave. A solution with two parallel tubes combined with heat spreaders and thermal vias is considered as a valid alternative and is also under study. The material budget of each plane is about $1.02 \% X_{0}$ according to the current stave design, where the sensor contributes for about $0.27 \% X_{0}$. The distribution of the radiation length, as a function of the pseudorapidity $\eta$, is reported in Fig. 6. 

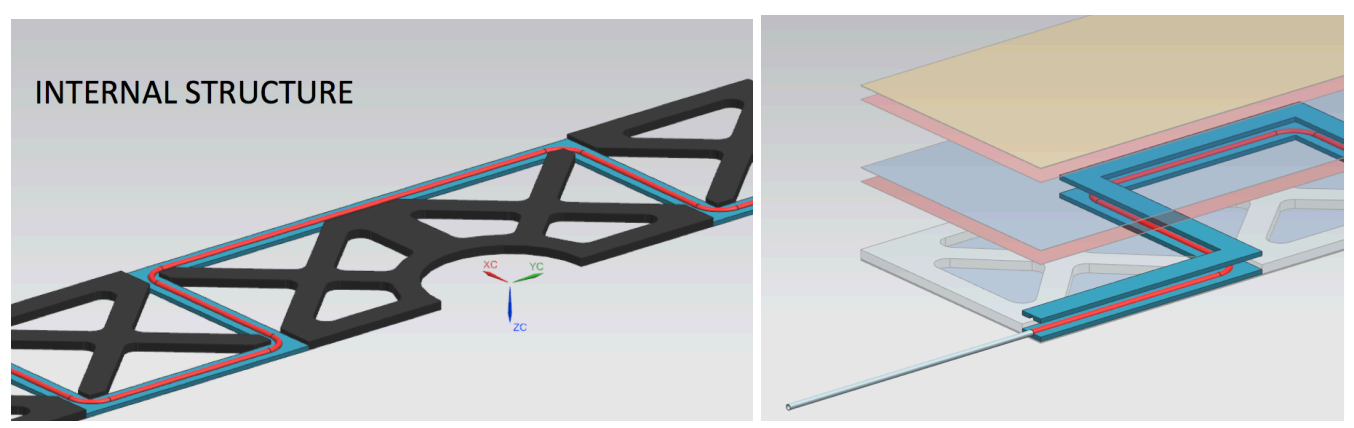

Figure 5: Left plot: the internal structure of the UT stave consists of the lightweight foam used for separating the CFRP facings and the carbon foam with the cooling tubes inside. Right plot: the titanium cooling pipes are embedded in the carbon foam and pass underneath the SALT chips for maximal heat transfer. The chips are positioned horizontally at the end of each hybrid module and a bent pipe ("snake pipe") provides maximal overlap.

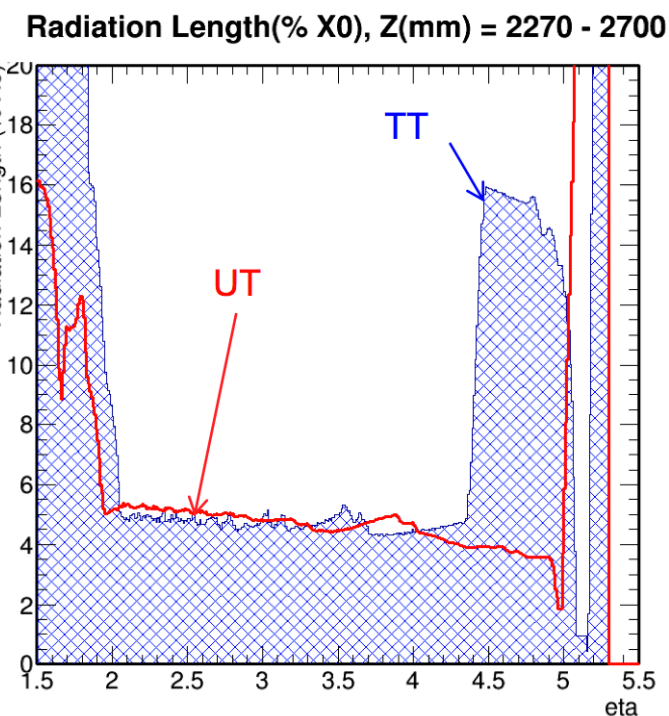

Figure 6: Radiation length $\left(\% X_{0}\right)$ as a function of the pseudorapidity $\eta$, between $z=2270-2700 \mathrm{~mm}$, for the UT design and the current TT detector. The calculation includes $0.14 \% X_{0}$ of air and $0.34 \% X_{0}$ for the detector box. Each plane contributes with $1.02 \% X_{0}$ to the total material budget. The reduction of material at $\eta=4.3-5.0$ is due to a new design of the beam pipe jacket based on aereogel and kapton.

\section{Conclusions}

We have discussed the conceptual design for the UT detector and its main objectives. UT is crucial detector for charged particle tracking and trigger decisions in the LHCb upgrade. It will be able to significantly reduce ghost tracks and provide useful information for the momentum measurement with about $15 \%$ precision in the $p_{\mathrm{T}}$ range $[0.1-10] \mathrm{GeV} / c$. In addition, an important speed up of trigger timings using VELO-UT momentum estimate (almost a factor 3 ) will be achievable. 
We are developing a complex and novel front end electronics device (SALT ASIC), and low mass high signal density cables to bring the electronics signal to the detector edge. The UT design features a $40 \mathrm{MHz}$ readout, an optimized segmentation of the sensors, a reduced material budget in the inner region of the detector due to a new beam pipe jacket design, and increased acceptance using circular cut-out sensors close to the beam pipe. The mechanics and the cooling are a challenge: the current design is inspired by the ATLAS IBL stave, with embedded $\mathrm{CO}_{2}$ evaporative cooling. The R\&D will continue until the Spring 2015 and we aim to be ready for installation in 2019.

\section{References}

[1] R. Aaij et al. (LHCb Collaboration), "Implications of LHCb measurements and future prospects," Eur. Phys. J. C 73, 2373 (2013).

[2] A. A. Alves, Jr. et al. (LHCb Collaboration), "The LHCb Detector at the LHC," JINST 3, S08005 (2008).

[3] C. Parkes, T. Szumlak, M. Idzik, M. Van Beuzekom, K. Wyllie, J. Buytaert, P. Collins and M. Artuso et al., "Preliminary Specification of a Silicon Strip Readout Chip for the LHCb Upgrade," LHCb-PUB-2012-011.

[4] K. Swientek, M. Firlej, T. Fiutowski, M. Idzik, J. Moron, T. Szmulak, "SALT - new silicon strip readout chip for the LHCb Upgrade," TWEPP2013, 23-27 September 2013, Perugia, Italy.

[5] M. Firlej, T. Fiutowski, M. Idzik, J. Moron, K. Swientek, "Development of scalable frequency and power Phase-Locked Loop (PLL) in 130nm CMOS technology," TWEPP2013, 23-27 September 2013, Perugia, Italy.

[6] The GBT project, https://espace.cern.ch/GBT-Project/

[7] R. Mountain et al., "Mechanical requirements document for the the LHCb upgrade UT tracker," private communication, October 2013.

[8] M. Cepeda et al., "Mechanical and Cooling Design Studies for an Integrated Stave Concept for Silicon Strip Detectors for the Super LHC," ATLAS-UPG-PUB-2008-001, 2008. 